\title{
Technikfolgenabschätzung soziotechnischer Zukünfte
}

\section{Ein Vorschlag zur wissenspolitischen Verortung des Vision Assessments}

\author{
Andreas Lösch, Institut für Technikfolgenabschätzung und Systemanalyse (ITAS), \\ Karlsruher Institut für Technologie (KIT), Karlstr. 11, 76133 Karlsruhe (andreas.loesch@kit.edu)
}

In Technikvisionen, Entwicklungsleitbildern und Zukunftsszenarien drücken sich Vorstellungen weitreichender „soziotechnischer Zukünfte“ aus, die vorgestellte Technikentwicklungen in Relation zu gesellschaftlichen Veränderungen stellen. Die Technikfolgenabschätzung (TA) stellen sie vor besondere analytische Herausforderungen, die nicht nur die Bewertung der Bedeutungen und Wirkungen dieser Zukunftsvorstellungen in Innovationsprozessen, sondern ebenso die eigene wissenspolitische Positionierung der TA betreffen. Ausgehend von den Forderungen des Diskussionspapiers „Technikfolgenabschätzung von soziotechnischen Zukünften“ zur Selbstverortung der TA wird ein Vorschlag gemacht, wie das Vision Assessment der TA seine eigene Rolle reflektieren kann, indem es seine Befassung mit soziotechnischen Zukünften in Forschung, Beratungen und Gestaltung als sozio-epistemische Praktiken begreift und sich wissenspolitisch ausrichtet.

\section{Technology assessment of sociotechnical futures}

A proposal for the scientific positioning of vision assessment in the field of knowledge politics

Visions of technology, guiding visions, and future scenarios are imaginaries of far-reaching "sociotechnical futures" that relate imagined technological developments to changes in society. They present particular analytical challenges to technology assessment (TA), concerning both the assessment of the meanings and impacts of these imaginaries in innovation processes and the self-reflexive positioning of TA itself within the field of knowledge politics. Answering to the demands of the discussion paper on "Technology assessment of sociotechnical futures" for a self-reflexive positioning of TA in the analyzed processes, a suggestion is made as to how TA's vision assessment can reflect upon its own role by understanding its own research, consulting and design of sociotechnical futures as socio-epistemic practices, positioning itself accordingly in the field of knowledge politics.

KEYWORDS: knowledge politics, reflexive technology assessment, socio-epistemic practices, sociotechnical futures, vision assessment

This is an article distributed under the terms of the Creative Commons Attribution License CCBY 4.0 (https://creativecommons.org/licenses/by/4.0/)

https://doi.org/10.14512/tatup.26.1-2.60

Eingereicht: 04. 04.2017. Angenommen: 30.05.2017

\section{Einleitung}

Um die Folgen von Technikentwicklungs- und Innovationsprozessen für Gesellschaft und Umwelt einzuschätzen, muss sich TA mit der Prozesswirksamkeit von Zukunftsvorstellungen befassen, die Technik- und Gesellschaftsentwicklung gleichermaßen betreffen. „Vision Assessment“ (Grin und Grunwald 2000; Lösch 2013) und „Hermeneutik der Technikzukünfte“ (Grunwald 2012, 2015) stellen Verfahren der TA dar, die fortlaufend weiterentwickelt werden, um die Bedeutungen und Wirkungen von Visionen, Entwicklungsleitbildern und Szenarien in gegenwärtig laufenden Innovations- und Transformationsprozessen untersuchen und bewerten zu können. Ziel dieser Forschungen ist die Erzeugung von Wissen, das über die Beratung entsprechender Adressaten, insbesondere über Politikberatung (Grunwald 2013), Entscheidungen orientiert und somit Interventionen in der Gestaltung der Prozesse (z. B. die Korrektur problematischer Verläufe) ermöglicht. Hierzu erzeugt die TA auch entsprechende Medien zur Entscheidungs- und Handlungsorientierung angesichts ungewisser Zukunft (wie etwa Szenarien und Roadmaps, z. B. Dieckhoff 2015; Schulz-Schaeffer 2013). Über ihre Beratungsfunktion interveniert die TA mit ihrem Vision Assessment in laufende Prozesse und ist immer auch Teil der ,Wissenspolitik“ (Stehr 2003) im jeweils tangierten Bereich der Gesellschaft. Doch wie können und sollen sich die TA und ihr Vision Assessment in den gesellschaftlichen Kontexten ihres Wirkens wissenspolitisch selbst verorten?

Das von dreiundzwanzig Autorinnen und Autoren aus TA und Science and Technology Studies (STS) kollektiv verfasste Diskussionspapier „Technikfolgenabschätzung von soziotechnischen Zukünften“ (Lösch et al. 2016) identifiziert aktuelle Defizite und drängende Herausforderungen der TA, einerseits bezüglich ihrer Selbstverortung als Akteur in den von ihr untersuchten Prozessen, andererseits bezüglich ihres Umgangs mit Zukunftsvorstellungen in Forschung, Beratung und Gestaltung. Gefordert wird: ,Die reflexive Befassung von TA mit soziotechnischen Zukünften erfordert eine Kontextualisierung der TA in den Konstellationen und Prozessen der Gegenwartsgesellschaft und ihrem Wandel, in denen sie selbst agiert.“ (Lösch et al. 2016, 
S. 14) Insgesamt liefert das Diskussionspapier vielseitige Begründungen dafür, warum und wie die TA die Gegenwart soziotechnischer Zukünfte (z. B. Technikvisionen, Leitbilder, Szenarien) untersuchen soll.

Mein Beitrag konzentriert sich speziell auf die Schlussfolgerungen des Diskussionspapiers zu Notwendigkeiten und Möglichkeiten einer neuen kontextuellen Selbstverortung der Befassung der TA mit soziotechnischen Zukünften (ebd., S. 1417). Um eine reflektierte wissenspolitische Positionierung der TA in den von ihr untersuchten und implizit wie explizit mitgestalteten Prozessen vornehmen zu können, schlage ich vor, das Analysekonzept „Leitbilder und Visionen als sozio-epistemi- wie kontextuellen Prozesswirksamkeiten von soziotechnischen Zukünften erforscht und bewertet, wirkt sie selbst - wie das Diskussionspapier ausführt - mit ihren Forschungs- und Beratungspraxen auf die untersuchten Prozesse ein und gestaltet diese mit. Dementsprechend sollte die TA nach dem Diskussionspapier auch die Bedeutung und Wirkmächtigkeit ihrer eigenen Befassung mit soziotechnischen Zukünften - als Akteur im untersuchten Geschehen - theoretisch, methodisch sowie kontextbezogen reflektieren und ausrichten (ebd., S. 14-17).

Die aktuelle Brisanz dieser Forderung gründet sich auf eine zweifache Gegenwartsdiagnose des Diskussionspapiers. Einerseits wird konstatiert, dass in den gesellschaftlichen und politi-

\section{Wenn TA die Inhalte sowie kontextuellen Prozesswirksamkeiten von soziotechnischen Zukünften erforscht und bewertet, wirkt sie selbst mit ihren Forschungs- und Beratungspraxen auf die untersuchten Prozesse ein und gestaltet diese mit.}

sche Praktiken“ des gleichnamigen Grundlagenforschungsprojekts am ITAS ${ }^{1}$ auf die Frage anzuwenden, wie sich die TA mit soziotechnischen Zukünften befasst und sich dabei selbstverortet? Auf dieser Ebene wird das Diskussionspapier weitergedacht.

\section{Diagnosen des Diskussionspapiers zur Selbstverortung der TA}

Unter soziotechnischen Zukünften werden alle technologiebezogenen Zukunftsvorstellungen und ihre Ausprägungen z. B. in Technikvisionen, Entwicklungsleitbildern und Zukunftsszenarien subsummiert. Die Betonung auf ,technologiebezogene Zukunftsvorstellungen“ (Lösch et al. 2016, S. 5) zeigt, dass der Begriff nichts mit tatsächlich eintretender Zukunft oder deren Prognose zu tun hat. Fokussiert wird auf in der Gegenwart, z. B. in Kommunikationsprozessen zirkulierende Zukunftsvorstellungen. In diesen drücken sich gegenwärtige Strukturen und Prozesse gesellschaftlicher Kontexte (z. B. Interessenskonstellationen, Organisationsformen, Machtverhältnisse) aus. Gleichermaßen sind die soziotechnischen Zukünfte in diesen Kontexten wirksam und gestalten diese mit (z. B. Entwicklungspfade, AgendaSetting). Von soziotechnischen Zukünften wird gesprochen, da diese Imaginationen immer technische Entwicklungen und soziale Veränderungen zueinander in Beziehung setzen. Die Reichweite der vorgestellten Veränderungen kann dabei sehr unterschiedlich sein (z. B. ein lokal begrenztes Innovationsprojekt oder die globale Energiewende). Wenn nun TA die Inhalte so-

1 https://www.itas.kit.edu/projekte_loes14_luv.php schen Kontexten des Agierens der TA seit einiger Zeit eine Ausweitung von Zukunftsdiskursen zu beobachten ist. In diesen stehen nicht mehr einzelne technische Entwicklungen im Zentrum (wie z. B. die Computer-Aided-Design-Technik). Die erweiterten Zukunftsdiskurse stellen Technik in Relation zu heterogenen gesellschaftlichen Einflussfaktoren und thematisieren Veränderungen weiter Teile der Gesellschaft (z. B. Energiewende, Industrie 4.0, Maßnahmen gegen den Klimawandel). Andererseits wird diagnostiziert, dass mit dieser Ausweitung der Zukunftsdiskurse eine Steigerung der Nachfragen nach entsprechenden Expertisen aus Politik und Gesellschaft sowie eine Vervielfältigung der mit soziotechnischen Zukünften befassten professionellen Akteure - neben TA z. B. Zukunftsforschung, Foresight, Innovationsstudien - einhergehe (ebd.).

Die Steigerung der Nachfrage hängt freilich auch mit der Bewältigung des immensen Nichtwissens zusammen, womit Auftraggeber und Adressaten der TA in ihrer Orientierung an den sich ausweitenden Zukunftsdiskursen konfrontiert werden $(\mathrm{Ku}-$ rath 2016). Die Befassung mit sehr weitreichenden soziotechnischen Zukünften erfordert das Zusammenspiel höchst unterschiedlicher Expertisen. TA kommt in diesem Netzwerk an unterschiedlichen Positionierungen und Standpunkten gar nicht umhin, sich mit ihrer Perspektive auf die jeweiligen soziotechnischen Zukünfte zu positionieren. Indem sie sich mit soziotechnischen Zukünften befasst, ko-moderiert und ko-moduliert sie, was diese ausdrücken und welche Wirkung diese in den Prozessen ihres Gebrauchs entfalten. Unübersehbar ist TA ein Akteur in den Prozessen, die sie selbst untersucht (ebd., S. 15-16; Rip 2012). Doch wie kann die TA die ihrer reflektierten wissenspolitischen Verortung in ihren Kontexten vorausgesetzte Selbstanalyse und Selbstbewertung durchführen? 
Soziotechnische Zukünfte als sozio-epistemische Praktiken

Das ITAS-Projekt „Leitbilder und Visionen als sozio-epistemische Praktiken" untersucht Funktionen und Wirkungen soziotechnischer Zukünfte - mit dem Fokus auf Visionen und Leitbilder - in gegenwärtig ablaufenden soziotechnischen Transformationsprozessen, aus denen zukünftige Innovationen hervorgehen können. Empirisch werden hierzu Praktiken untersucht, wie z.B. forschungspolitische Aushandlungen, die Erstellung von Szenarien und Roadmaps, Realexperimente, Mediendebatten und Laborkommunikation, in denen sich die Neuordnungen bestehender sozialer und technischer Konstellationen vollziehen. Diese sind dem Voranschreiten übergreifender Transformationsprozesse (z. B. Energiewende, Digitalisierung der Gesellschaft) vorausgesetzt. Ein Beispiel sind etwa die Neuordnungen der soziotechnischen Konstellationen zwischen Prozessen, Akteuren oder Techniken des Energiesystems durch seine Dezentralisierungen und Digitalisierungen, die im Zuge der Energiewende in Realexperimenten zur Implementierung von Smart-Grid-Designs erprobt werden (Lösch und Schneider 2016). Das Forschungsinteresse des ITAS-Projekts liegt auf der Untersuchung der Funktionen und Wirkungen, die „,soziotechnische Zukünfte“ innerhalb dieser Praktiken der Neuordnung haben. Durch den Fokus auf die empirische Erfassung von Funktionen und Wirkungen soziotechnischer Zukünfte in für Prozessverläufe konstitutiven Praktiken, wird für die TA eine Untersuchung und Bewertung von Leitbildern, Visionen, Szenarien und anderen Medien soziotechnischer Zukünfte im Kontext sich wandelnder wissenschaftlicher, technischer und sozialer Bedingungen möglich. Das heißt, in Weiterentwicklung bisheriger Ansätze des Vision Assessments der TA, sind nicht nur die Inhalte, Versprechen oder Narrative von soziotechnischen Zukünften vor dem Hintergrund gegenwärtiger wissenschaftlicher, technischer und sozialer Bedingungen (z. B. hinsichtlich ihrer Realisierbarkeit und Wünschbarkeit) bewertbar. Vielmehr werden die Folgen soziotechnischer Zukünfte auf Prozessverläufe einschätzbar. Die Leitfrage ist nicht, ob die Prognosen und Versprechen der Visionen realistisch und wünschenswert sind, sondern, was soziotechnische Zukünfte (wie z. B. Visionen und Leitbilder) in laufenden Transformationsprozessen der Gegenwart ermöglichen und ob die gegenwärtig beobachtbaren soziotechnischen Effekte (z. B. Digitalisierung funktionaler Interaktionen der Governance des Energiesystems, Hochtechnisierung von Funktionen von Pflegearrangements, Roboterisierung der Arbeit im Zeichen von Industrie 4.0) wünschenswert sind. Dieses konstellations- und prozessbezogene Wissen soll wissenspolitische Interventionen der TA in Prozesse durch ihre Beratungspraxen fundieren.

Orientierend für eine solche Prozess- und Praxisperspektive des Vision Assessments sind sozialwissenschaftliche STSForschungen zu Zukunftsbildern, kollektiven Erwartungen, Utopien und antizipativen Praktiken in Innovationsprozessen (z. B. van Lente 1993; Brown et al 2000; Konrad et al. 2016; Dickel und Schrape 2017). Das ITAS-Projekt will erfassen, was Vi- sionen in Arrangements heterogener Elemente (wie Visionen, Akteure, Dinge, Organisationen) ermöglichen. Solche Arrangements lassen sich in jedem der untersuchten prozessrelevanten Kontexte der Gesellschaft (z. B. Labor, Forschungspolitik, Realexperiment) rekonstruieren. Visionen werden dabei als sozioepistemische Praktiken untersucht. Damit werden Praktiken bezeichnet, die gleichzeitig das neue Wissen und die sozialen Arrangements erzeugen, die Transformationsprozesse ermöglichen und vorantreiben. Um die praktischen Funktionen und Wirkungen von soziotechnischen Zukünften untersuchen zu können, werden im Analysekonzept des Projekts vier funktionale Dimensionen von Visionen als sozio-epistemische Praktiken unterschieden. Im Rahmen des Projektes werden sie in ein analytisches Konzept integriert. Erst ihr Zusammenwirken lässt eine soziotechnische Zukunft praktisch in Prozessen wirksam werden.

1. Visionen wirken als sozio-epistemische Praktiken, indem sie diskursive Schnittstellen zwischen Gegenwart und Zukunft, das heißt zwischen gegenwärtigen Zuständen, Entwicklungen und zukünftigen Problemlösungen konstruieren (Adams und Groves 2007; Brown et al 2000). Hierdurch produzieren sie entscheidungs- und handlungsorientierendes Wissen, sowohl für diejenigen Akteure, die den Lösungsangeboten folgen, als auch für diejenigen, die diese Problemlösungen ablehnen oder kritisieren.

2. Visionen sind Kommunikationsmedien (Lösch 2014, S. 56 ff., $72 \mathrm{ff}$.). Die durch Visionen entworfenen Zukünfte lassen sich unterschiedlich interpretieren. Über die visionären Problemlösungen kann verhandelt und gestritten werden (Brown et al. 2000). In der Auseinandersetzung mit bestimmten Visionen in spezifischen Praxisfeldern treffen Akteure mit unterschiedlichen Perspektiven aufeinander. Eine Vision konstituiert einen Kommunikationsprozess, der zu neuen Kooperationen sowie zu Friktionen zwischen den beteiligten Akteuren führen kann.

3. Visionen können die Koordination von Akteuren und ihren Praktiken untereinander ermöglichen (Dierkes et al. 1992; Böhle und Bopp 2014). Eine Vision kann ein praxiswirksames Leitbild sein, das durch seine Orientierungsfunktion zur Umformung bestehender oder zur Etablierung neuer soziotechnischer Konstellationen beiträgt.

4. Visionen entfalten in der Praxis eine aktivierende Macht auf die mit ihnen konfrontierten Akteure. Indem die Vision ihr Problemlösungsangebot als unhinterfragt notwendig und wünschenswert positioniert, fordert sie zum Mitwirken an der Zielerreichung auf (z. B. Jasanoff und Kim 2015; Nordmann 2010). Entweder haben die adressierten Akteure an der Realisierung des Ziels mitzuwirken oder sie sind genötigt, alternative soziotechnische Zukünfte und Lösungsangebote zu entwerfen.

Diese Wirkungen von Visionen als sozio-epistemische Praktiken auf Umordnungen soziotechnischer Konstellationen lassen sich mit Hilfe des Analysekonzepts in Fallstudien des Projekt- 
teams untersuchen. Am Beispiel technikdeterministischer Visionen einer digitalen Berechenbarkeit der Welt in politischen und öffentlichen Debatten zu Big Data zeigt sich, wie unterschiedliche Akteure der Forschung, in Unternehmen und tendenziell alle Bürgerinnen und Bürger nicht nur für das Sammeln von Daten, sondern auch für das Integrieren der Daten verantwortlich gemacht und aktiviert werden (z. B. Lösch, Heil und Schneider im Druck). Die Analyse des Gebrauchs von Visionen des Smart Grids in Feldexperimenten rekonstruiert, wie mittels der Vision neue Rollen und Interaktionen zwischen Stromanbietern, Dienstleistern, Konsumenten, Regularien, Kraftwerken, Informationstechnologien und Märkten erprobt werden. Deutlich wird, dass ein durch Smart Grids kontrolliertes und dezentralisiertes Energiesystem der Zukunft eine grundlegende Neuordnung der für das Energiesystem konstitutiven soziotechnischen Konstellationen und damit der etablierten Macht- und Rollenverteilungen erforderlich macht (Lösch und Schneider 2016). Am Beispiel von In-vitro-Fleisch wird einsichtig, wie eine Vision, die das Laborprodukt in eine Maßnahme zur Lösung von Problemen des Klimawandels übersetzt, zur Herausbildung neuer sozialer Allianzen und strategischer Kooperationen zwischen Umwelt- und Tierschutzverbänden, Forschungseinrichtungen, Innovatoren und Lebensmittelproduzenten führen kann (Ferrari und Lösch 2017). Anhand der Herausbildung von Organisationsformen der Open-Digital-Fabrikation lässt sich darstellen, wie das Zusammenwirken von Visionen der Openness, technischen $\mathrm{Ob}$ jekten und involvierten Akteuren zu unterschiedlichsten organisationalen Ausprägungen sogenannter Fab Labs führt (vgl. Schneider 2017). In allen Fällen handelt es sich um gegenwärtig laufende Transformationsprozesse mit heute noch ungewissem Ausgang. Dementsprechend ermöglicht das Analysekonzept dem Vision Assessment in den Fallstudien eine Prozessverlaufsbeobachtung. Gegenwärtige Entwicklungen wurden identifizierbar, kritisierbar und können über die Beratung entsprechender politischer und gesellschaftlicher Instanzen potenziell nachjustierbar werden. Doch wie wirkt das Vision Assessment der TA an den untersuchten Praktiken und Prozessen selbst mit?

\section{TA als Akteur und Vision Assessment als sozio-epistemische Praktik}

Wendet man das analytische Konzept auf die TA und ihre Vision Assessments an, dann ist die Befassung der TA mit soziotechnischen Zukünften selbst eine Praktik, durch die Visionen als sozio-epistemische Praktiken wirksam werden. Die jeweilige Vision, mit der sich TA befasst, stellt auch für die TA eine Schnittstelle zwischen Zukunft und Gegenwart dar, deren Problemlösungsangebote die TA beurteilt und dem gegenüber die TA gegebenenfalls Alternativlösungen formuliert (z. B. kritisch durch das Identifizieren überzogener Versprechen oder von Engführungen, die erwünschte Innovationswege ausschließen). Da die TA eine beratende Wissenschaft ist und sich mit anderen Akteuren über die Vision verständigt, ist eine Vision auch für sie ein Kommunikationsmedium, das Verständigungen und bisweilen Kontroversen mit anderen Akteuren ermöglicht. Die Assessments der TA können zu Produktionen von neuem Wissen über das Medium der Vision und zur Herausbildung neuer sozialer Arrangements beitragen, die sich auch in neuen Formen der Koordination und Governance von Prozessen im jeweiligen Praxisfeld niederschlagen können. In dieses ist die TA als Akteur involviert - beispielsweise durch forschungspolitische Beratung, projektintegrierte Entwicklungsmitgestaltung als constructive TA (CTA), Deliberation in partizipativen Verfahren oder als politikrelevante wissenschaftliche Expertise zur Identifizierung

\section{Als sozio-epistemische Praktik}

\section{ist TA an der praktischen Erzeugung des Wissens und der sozialen Arrangements beteiligt.}

von Regulierungsbedarfen. Entscheidend ist, dass die TA in ihrer Befassung mit einer Vision selbst zur Positionierung gegenüber den visionären Versprechen aktiviert wird und dass sie mit ihren Expertisen wiederum andere Akteure zur Stellungnahme aktiviert. TA ist damit selbst Teil der Prozesse, die sie mit ihren Vision Assessments in den Blick nimmt. Als sozio-epistemische Praktik ist sie an der praktischen Erzeugung des Wissens und der sozialen Arrangements beteiligt, die für die Prozesse konstitutiv sind.

Diese Funktion und Wirkung der TA in ihren Forschungs-, Beratungs- und Gestaltungspraktiken auch selbst zu reflektieren, ist den Diagnosen des Diskussionspapiers zufolge jedoch kein leichtes Geschäft (Lösch et al. 2016). Vision Assessments zu räumlich und zeitlich sehr weitreichenden soziotechnischen Zukünften (z. B. globale Energiewende, Klimawandel, Digitalisierung der Gesellschaft) in entsprechenden Transformationsprozessen kann die TA nur in Kooperation mit anderen Expertiseanbietern (z. B. aus Foresight, STS-Forschung, Innovationsforschung) und durch Berücksichtigung von deren Wissensbeständen, Theorien und Methoden durchführen. Zudem hat die TA das Wissen und die Einschätzungen jener gesellschaftlichen Akteure mit einzubeziehen, die sie beraten will Wissen, das auch erst im Beratungsprozess entsteht (ebd., S. 1617). Durch die Reichweite der Zukunftsdiskurse kann die TA auch nur ein perspektivisches und standortgebundenes Wissen in Bezug auf all die sozialen und technischen Transformationen produzieren, die mit den soziotechnischen Zukünften adressiert werden. Jedes Vision Assessment ist damit eine sozio-epistemische Praktik. Zusammen mit den Praktiken anderer professioneller Akteure zu derselben soziotechnischen Zukunft, z. B. in der Forschungspolitik, in der Entwicklung von Szenarien, in den Medien, bringt das Vision Assessment erst das Wissen her- 
vor und trägt zur Bildung der sozialen Arrangements miteinander kooperierender Akteure bei, die für die Transformationsprozesse konstitutiv sind. In Wechselwirkung mit anderen sozio-epistemischen Praktiken und ihren jeweiligen Varianten soziotechnischer Zukünfte beeinflusst TA folglich aktiv und gestaltend den Verlauf von Innovationsprozessen.

\section{Zu Chancen wissenspolitischer Selbstverortung}

Wenn sich TA also selbst als Akteur in den übergreifenden Prozessen reflektiert, in denen soziotechnische Zukünfte moduliert und dadurch verändert werden, kann sie sich nicht auf die Position eines kritischen Beobachters zurückziehen. Wie das Diskussionspapier ausführt, positioniert sie sich und wird positioniert zwischen Förderung und Kritik soziotechnischer Zukünfte, die für die Transformation der Gesellschaft oder zumindest gesellschaftlicher Bereiche konstitutiv sind (Lösch et al. 2016, S. 15). Angesichts dieser Lage ergeben sich nach dem Diskussionspapier für die TA als Forschung weitreichende Kooperationsbedarfe mit diversen Geistes-, Sozial- und Kulturwissenschaften. Für die TA als Beratung ist nicht nur eine Spezifizierung der Angebote je nach Adressaten, sondern auch eine Umgestaltung des Beratungsprozesses dahingehend nötig, dass die Beratenen in die Wissensproduktion integriert werden. Für explizite Gestaltungspraktiken der TA ist eine systematische Integration der Kontext- und Prozessperspektive in ihre Praktiken etwa der Entwicklung von Szenarien, CTA in Entwicklungsprojekten, partizipativer TA, Visioneering in Foresightpraktiken etc. - erforderlich. Der „hohe“ Anspruch lautet, dass sich TA in den Prozessen, die sie untersucht und dabei mitgestaltet reflektiert zu positionieren habe und dabei zugleich immer die Wirkungen ihres Tuns bzw. die Wechselwirkung ihres Tuns mit dem anderer Akteure zu berücksichtigen sei. Dass dies vollständig unmöglich ist, sollte mit den Ausführungen dieses Beitrags deutlich geworden sein. Dass aber ein Rückzug auf eine distanzierte und nach wissenschaftlichen und sozialen Gütekriterien durchgeführte TA-Praxis auch unzureichend ist, sollte ebenso deutlich geworden sein.

Eine Chance zur Reflexion, Positionierung und Ausjustierung des eigenen Tuns bietet die vorgeschlagene Selbstanwendung des Analysekonzepts von Visionen als sozio-epistemische Praktiken. Mit diesem Raster kann TA an den konkreten und lokalen Orten ihres Wirkens - beispielsweise als Teilnehmerin oder Moderatorin in Prozessen des forschungspolitischen Agenda-Settings, in Partizipationsverfahren, in Realexperimenten - identifizieren, welche Folgerungen sie selbst wie andere beteiligte Akteure aus den Problemlösungsangeboten einer soziotechnischen Zukunft (Schnittstellenfunktion) zieht, welche Kommunikationseffekte daraus entstehen (Kommunikationsmedium), welche Kooperationsformen sich zwischen unterschiedlichen Akteuren bilden (Koordinationsfunktion) und wie sie oder andere Akteure durch die normativen Implikationen der sozio- technischen Zukünfte zur Mitwirkung an den sozio-epistemischen Praktiken aufgefordert werden (Aktivierungsfunktion). Indem TA diese Funktionen im Blick hat, wird sie auch befähigt, strategisch in Prozesse $\mathrm{zu}$ intervenieren und diese ausgehend vom jeweiligen Praxisfeld mitzugestalten. Die weitreichenden Effekte der eigenen Praxis auf Gesamtprozesse der Transformation kann sie freilich nicht kennen. Aber sie kann sich - gemäß den Forderungen des Diskussionspapiers - als eine kontext- und prozessreflexive wissenspolitische Praxis positionieren.

\section{Eine mit soziotechnischen Zukünften befasste TA ist immer auch eine Gestaltungswissenschaft.}

Im Bewusstsein, dass jedes Vision Assessment auch die Wirkung einer Vision mit befördert, muss sich eine ihrer wissenspolitischen Lage bewusste TA der soziotechnischen Zukünfte auch als eine „Visioneering TA“ (Schneider und Lösch 2015) begreifen, die als kritischer Akt auch eigene Visionen entwirft, anstatt nur die Visionen zu kritisieren oder zu befördern, die bereits im Spiel sind. Eine mit soziotechnischen Zukünften befasste TA ist immer auch eine Gestaltungswissenschaft. Sie kann und sollte ihre Standpunkte und Praktiken im Gestaltungsprozess aber nachvollziehbar vermitteln.

\section{Danksagung}

Ich danke für die hilfreichen Kommentare zur Fertigstellung dieses Artikels aus den beiden Gutachten und aus meinem Projektteam.

\section{Literatur}

Adam, Barbara; Groves; Chris (2007): Future Matters: Action, Knowledge, Ethics. Boston: Brill.

Böhle, Knud; Bopp, Kolja (2014): What a Vision: The Artificial Companion A Piece of Vision Assessment Including an Expert Survey. In: Science, Technology \& Innovation Studies 10 (1), S. 155-186.

Brown, Nik; Rappert, Brian; Webster, Andrew (Hg.) (2000): Contested Futures: A Sociology of Prospective Techno-science. Burlington: Ashgate.

Dickel, Sascha; Schrape, Jan-Felix (2017): The Logic of Digital Utopianism. In: Nanoethics 11, S. 47-58.

Dieckhoff, Christian (2015): Modellierte Zukunft - Energieszenarien in der wissenschaftlichen Politikberatung. Bielefeld: Transkript.

Dierkes, Meinolf; Hoffmann, Ute; Marz, Lutz (1992): Leitbild und Technik. Zur Entstehung und Steuerung technischer Innovationen. Berlin: Edition Sigma. Ferrari, Arianna; Lösch, Andreas (2017): How Smart Grid Meets In Vitro Meat: on Visions as Socio-Epistemic Practices. Nanoethics 11, S. 75-91.

Grin, John; Grunwald, Armin (2000): Vision Assessment: Shaping Technology in 215t Century Society. Towards a Repertoire for Technology Assessment. Berlin/New York: Springer. 
Grunwald, Armin (2012): Technikzukünfte als Medium von Zukunftsdebatten und Technikgestaltung. Karlsruhe: KIT Scientific Publishing.

Grunwald, Armin (2013): Techno-visionary Sciences. Challenges to Policy Advice. In: Science, Technology \& Innovation Studies 9 (2), S. 21-38.

Grunwald, Armin (2015): Die hermeneutische Erweiterung der Technikfolgenabschätzung. In: Technikfolgenabschätzung - Theorie und Praxis 24 (2), S. 65-69.

Jasanoff, Sheila; Kim, Sang-Hyun (Hg.) (2015): Dreamscapes of Modernity. Sociotechnical Imaginaries and the Fabrication of Power. Chicago: University of Chicago.

Konrad, Konrad; van Lente, Harro; Groves Chris; Selin, Cynthia (2016). Performing and Governing the Future in Science and Technology. In: Felt, Ulrike; Fouché, Rayvon; Miller, Clark A.; Smith-Doerr, Laurel (Hg.): The Handbook of Science and Technology Studies, $4^{\text {th }}$ edition. Cambridge: MIT Press, S. 465-493.

Kurath, Monika (2016): Nichtwissen lenken. Nanotechnologie in Europa und den Vereinigten Staaten. Baden-Baden: Nomos.

Lösch, Andreas (2013): Vision Assessment zu Human-Enhancement-Technologien. Konzeptionelle Überlegungen zu einer Analytik von Visionen im Kontext gesellschaftlicher Kommunikationsprozesse. In: Technikfolgenabschätzung Theorie und Praxis 22 (1), S. 9-16.

Lösch, Andreas (2014): Die diskursive Konstruktion einer Technowissenschaft. Wissenssoziologische Analytik am Beispiel der Nanotechnologie. BadenBaden: Nomos.

Lösch, Andreas et al. (2016): Technikfolgenabschätzung von soziotechnischen Zukünften. Karlsruhe: KIT - ITZ Diskussionspapiere, Nr. 03, 2016. Online verfügbar unter: https://www.itz.kit.edu/112.php, zuletzt geprüft am 30. 05.2017

Lösch, Andreas; Heil, Reinhard; Schneider Christoph (im Druck): Responsibilization through Visions. In: Journal of Responsible Innovation (angenommen am 19.05.2017)

Lösch, Andreas; Schneider, Christoph (2016): Transforming Power/Knowledge Apparatuses: the Smart Grid in the German Energy Transition. In: Innovation: The European Journal of Social Science Research 29 (3), S. 262-284.
Nordmann, Alfred (2010): A Forensics of Wishing: Technology Assessment in the Age of Technoscience. In: Poiesis \& Praxis 7 (1), S. 5-15.

Rip, Arie (2012): Futures of Technology Assessment. In: Decker, Michael; Grunwald, Armin; Knapp, Martin (Hg.): Der Systemblick auf Innovation. Technikfolgenabschätzung in der Technikgestaltung. Berlin: Edition Sigma, S. 29-42.

Schneider, Christoph (2017): Transforming TechKnowledgies: The Case of Open Digital Fabrication. München: Univ. Diss. (angenommen März 2017).

Schneider, Christoph; Lösch, Andreas (2015): What About Your Futures, Technology Assessment? An Essay on How to Take the Visions of TA Seriously, Motivated by the PACITA Conference. In: Technikfolgenabschätzung - Theorie und Praxis 24 (2), S. 70-74.

Schulz-Schaeffer, Ingo (2013): Scenarios as Patterns of Orientation in Technology Development and Technology Assessment. Outline of a Research Program. In: Science, Technology \& Innovation Studies 9 (1), S. 23-44.

Stehr, Nico (2003): Wissenspolitik. Die Überwachung des Wissens. Frankfurt am Main: Suhrkamp.

Van Lente, Harro (1993): Promising Technology. The Dynamics of Expectations in Technological Developments. Enschede: Univ. Diss. Twente.

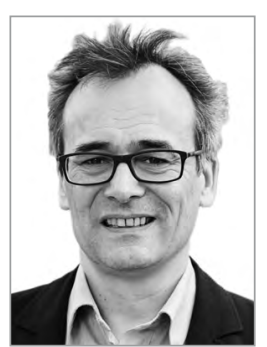

\section{PD DR. ANDREAS LÖSCH}

ist Wissens- und Techniksoziologe mit Fokus auf Science \& Technology Studies. Als Senior Research Scientist im FB „Wissensgesellschaft und Wissenspolitik“ des ITAS/KIT leitet er das Projekt „Leitbilder und Visionen als sozio-epistemische Praktiken". Diverse Publikationen u. a. zu Vision Assessment und Diskursforschung.

\section{politische ökologie}

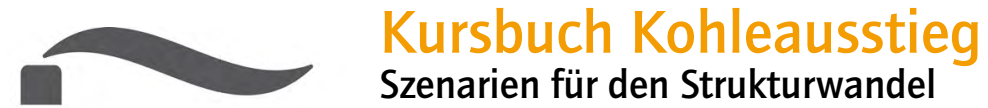

Angesichts des voranschreitenden Klimawandels kommt Schwung in die politische Debatte um die wahren ökologischen und sozialen Kosten der Kohleverbrennung. Auch Deutschland wird restlos aus der Kohleverstromung aussteigen müssen. Anders lassen sich die Ziele des Pariser Klimaabkommens nicht erfüllen. Zum Glück liegen längst konkrete Vorschläge für einen sozialverträglichen Kohleausstieg auf dem Tisch. Wenn die nächste Bundesregierung sie aufgreift, könnten sich Deutschlands Braunkohlegebiete zu Reallaboren einer postfossilen Wirtschaft und Gesellschaft entwickeln.

Mitherausgegeben von der European Climate Foundation. Mit Beiträgen von Felix Chr. Matthes, Karsten Smid, Gerd Rosenkranz, Svenja Künstler, Martin Rocholl, Charlotte Loreck, Dirk Jansen u.v.m. 